\title{
New Iboga-Type Indole Alkaloids from Tabernaemontana divaricata
}

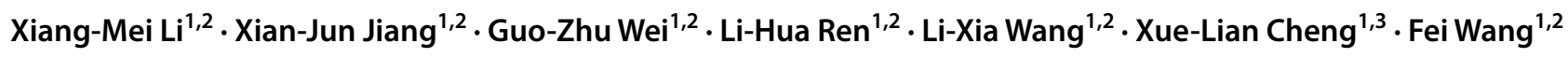

Received: 27 August 2019 / Accepted: 5 November 2019 / Published online: 14 November 2019

(c) The Author(s) 2019

\begin{abstract}
Two hitherto unknown iboga-type indole alkaloids, namely $(3 R)$-7,19-di-epi-3-methoxytabernoxidine (1) and $(3 R, 19 R)$ 19-hydroxy-3-(2-oxopropyl)voacangine (2), together with eight known alkaloids (3-10), were isolated from the twigs and leaves of Tabernaemontana divaricata. Their structures were established on the basis of spectroscopic data interpretation, single crystal X-ray diffraction analysis and circular dichroism spectrum.
\end{abstract}

\section{Graphic Abstract}

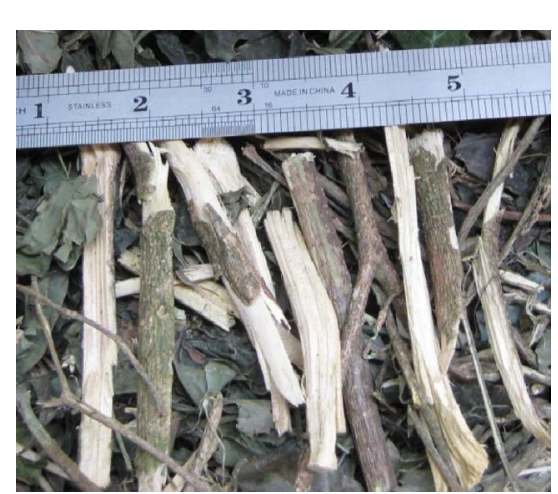

Tabernaemontana divaricata
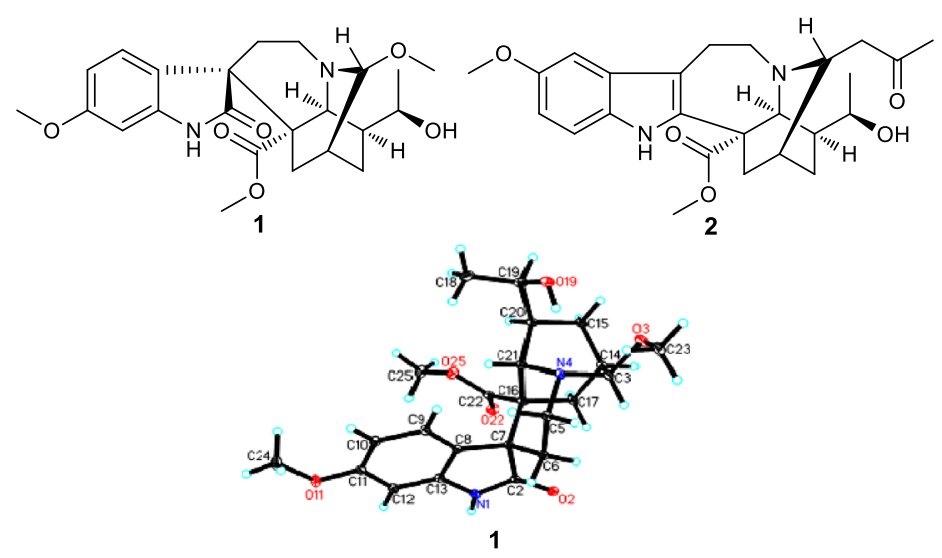

Keywords Tabernaemontana divaricata $\cdot$ Iboga-type indole alkaloid $\cdot$ Single crystal X-ray diffraction

\section{Introduction}

The genus Tabernaemontana (Apocynaceae family), comprising 120 species, is a rich source of monoterpenoid indole alkaloids with diverse skeletons, particularly iboga-type alkaloids [1-3]. Tabernaemontana divaricata, distributed in Yunnan and Guangxi Provinces of China [4], is widely used as folk medicine for the treatment of sore throat and hypertension [5], and a series of monoterpenoid indole and bisindole alkaloids were reported [6,7]. As part of a BioBioPha (https://www.chemlib.cn) objective to assemble a large-scale natural product library valuable in the discovery of new drug leads from nature, the phytochemical investigation on the twigs and leaves of $T$. divaricata resulted in the isolation of two new iboga-type indole alkaloids, namely (3R)-7,19-di-epi-3-methoxytabernoxidine 
(1) and $(3 R, 19 R)$-19-hydroxy-3-(2-oxopropyl)voacangine (2) (Fig. 1), together with eight known indole alkaloids: 19,20-(E)-vallesamine (3) [8, 9], ibogaine (4) [10], conophylline (5) [11], taberdivarine $\mathrm{H}$ (6) [12], conodurine (7) [13], hecubine (8) [14], voafinidine (9) [15], and tabernanthine $(\mathbf{1 0})[10,16]$. In this paper, we report the isolation and structure elucidation of new alkaloids $\mathbf{1}$ and $\mathbf{2}$.

\section{Results and Discussion}

Compound 1, obtained as colorless block crystals from $\mathrm{MeOH}$, possessed a molecular formula of $\mathrm{C}_{23} \mathrm{H}_{30} \mathrm{~N}_{2} \mathrm{O}_{6}$, as evidenced by HR-ESI-MS (pos.) at $\mathrm{m} / \mathrm{z} 453.1997$ $[\mathrm{M}+\mathrm{Na}]^{+}$(calcd. for $\mathrm{C}_{23} \mathrm{H}_{30} \mathrm{~N}_{2} \mathrm{O}_{6} \mathrm{Na}$, 453.1996), requiring 10 degrees of unsaturation. The ${ }^{1} \mathrm{H}$ NMR spectrum in chloroform- $d$ (Table 1) displayed one $\mathrm{NH}$ proton at $\delta_{\mathrm{H}}$ $7.90(1 \mathrm{H}, \mathrm{s})$, one 1,2,4-trisubstituted benzene ring at $\delta_{\mathrm{H}}$ $7.63(1 \mathrm{H}, \mathrm{d}, J=8.5 \mathrm{~Hz}), 6.54(1 \mathrm{H}, \mathrm{dd}, J=8.5,2.6 \mathrm{~Hz})$, and $6.43(1 \mathrm{H}, \mathrm{d}, J=2.6 \mathrm{~Hz})$, two oxygenated methines at $\delta_{\mathrm{H}} 4.18(1 \mathrm{H}, \mathrm{d}, J=3.3 \mathrm{~Hz})$ and $3.95(1 \mathrm{H}, \mathrm{m})$, one secondary methyl at $\delta_{\mathrm{H}} 1.24(3 \mathrm{H}, \mathrm{d}, J=6.4 \mathrm{~Hz})$, and three methoxy groups at $\delta_{\mathrm{H}} 3.78,3.33$, and 3.19 (each $3 \mathrm{H}, \mathrm{s}$ ). The ${ }^{13} \mathrm{C}$ NMR spectrum (Table 1) displayed a total of 23 carbon resonances, including two overlapping signals (C-5 and C-16). Combined with the DEPT spectrum, different types of carbons were distinguished to be four methyls, four methylenes, eight methines and seven quaternary carbons (including two carbonyls at $\delta_{\mathrm{C}} 179.2$ and 174.3). The HSQC and ${ }^{1} \mathrm{H}-{ }^{1} \mathrm{H}$ COSY spectra (Fig. 2) revealed the connections of C-3/C-14 and C-17/C-14/C-15/C-20/C19/C-18, which were confirmed by the HMBC correlations (Fig. 2) from $\mathrm{H}-3$ to $\mathrm{C}-15$ and $\mathrm{C}-17, \mathrm{H}-15$ to $\mathrm{C}-17$ and $\mathrm{C}-19$, and Me-18 to C-19 and C-20. The above NMR analysis indicated the planar structure of $\mathbf{1}$ was remarkably similar to that of tabernoxidine (Fig. 1) [17], an iboga-type spiro oxindole alkaloid whose absolute stereochemistry has been established by single crystal X-ray diffraction. The prominent difference between them was the absence of resonance for a methylene (C-3) and the presence of resonances for a downfield oxygenated methine and a methoxy. The oxygenated methine was assigned to C-3 based on the HMBC correlations from $\mathrm{H}-21$ to $\mathrm{C}-3$, and from $\mathrm{H}-3$ to $\mathrm{C}-5, \mathrm{C}-15$, and $\mathrm{C}-17$. And the methoxy was positioned at $\mathrm{C}-3$ by the HMBC correlation between the methoxy protons and C-3. In the ROESY spectrum (Fig. 3), the correlations between $\mathrm{H}-9$ and $\mathrm{H}-21 / \mathrm{H}-5 \alpha$ revealed $R^{*}$ configuration of the spiro carbon atom at $\mathrm{C}-7$, and the correlations between $\mathrm{H}-3$ and $\mathrm{H}-5 \beta / \mathrm{H}-6 \beta / \mathrm{H}-17 \beta$ indicated $R^{*}$ configuration of $\mathrm{C}-3$. The above deduction was confirmed by single crystal $\mathrm{X}$-ray diffraction using Mo $\mathrm{K} \alpha$ radiation (Fig. 4). Since the absolute stereochemistry of iboga-type indole alkaloids from Apocynaceae had been determined previously by single crystal X-ray diffraction using $\mathrm{Cu} \mathrm{K} \alpha$ radiation or electronic circular dichroism (ECD) analysis $[1-3,17]$, biogenetically, the absolute configuration of $\mathbf{1}$ could be deduced as $3 R, 7 R, 14 R, 16 R, 19 R, 20 S, 21 S$. Therefore, the structure of $\mathbf{1}$ was established and named as $(3 R)$ 7,19-di-epi-3-methoxytabernoxidine, as shown in Fig. 1.

Compound 2, white amorphous powder, gave a molecular formula of $\mathrm{C}_{25} \mathrm{H}_{32} \mathrm{~N}_{2} \mathrm{O}_{5}$ as determined by positive HRESIMS at $\mathrm{m} / z 441.2384[\mathrm{M}+\mathrm{H}]^{+}$(calcd. for $\mathrm{C}_{25} \mathrm{H}_{33} \mathrm{~N}_{2} \mathrm{O}_{5}, 441.2384$ ) with 11 degrees of unsaturation. The ${ }^{1} \mathrm{H}$ and ${ }^{13} \mathrm{C}$ NMR spectra (Table 1 ) showed that $\mathbf{2}$ had a structure similar to that of 3-(2-oxopropyl)voacangine [18], except for the presence of a 1-hydroxyethyl group $\left[\delta_{\mathrm{H}}\right.$ $3.89(1 \mathrm{H}, \mathrm{qd}, J=6.5,2.0 \mathrm{~Hz}) / \delta_{\mathrm{C}} 71.0(\mathrm{~d})$ and $\delta_{\mathrm{H}} 1.28(3 \mathrm{H}$, $\left.\mathrm{d}, J=6.5 \mathrm{~Hz}) / \delta_{\mathrm{C}} 22.2(\mathrm{q})\right]$ in place of an ethyl side chain at $\mathrm{C}-20$, which was confirmed by the HMBC correlations (Fig. 2) from Me-18 to C-19 and C-20. The ROESY correlations (Fig. 3) of H-21 $\leftrightarrow \mathrm{H}-5 \alpha / \mathrm{H}-6 \alpha, \mathrm{H}-17 \alpha \leftrightarrow \mathrm{H}-20 / \mathrm{H}-$ $15 \alpha$ suggested that these protons were $\alpha$-oriented, and the correlations of $\mathrm{H}-3 \leftrightarrow \mathrm{H}-17 \beta, \mathrm{H}-1^{\prime} \leftrightarrow \mathrm{H}-15 \beta$ revealed the stereochemistry of $\mathrm{C}-3$, which indicated that the configurations of C-3, C-14, C-16, C-20 and C-21 were identical to those in 3-(2-oxopropyl)voacangine. Comparison of the ${ }^{13} \mathrm{C}$ NMR chemical shifts of $\mathrm{C}-15$ and $\mathrm{C}-21$ as well as the ${ }^{1} \mathrm{H}$ NMR chemical shift of $\mathrm{H}-19$ with those of $19 R / S$ hydroxyiboga alkaloids indicated a $19 R$ configuration $[19$, 20]. Additionally, the Cotton effects in the CD spectrum (See S14, Supplementary Material) of 2 were in good agreement with those for voacangine [18], so the absolute configuration of 2 was assigned as $3 R, 14 R, 16 S, 19 R, 20 S, 2$ $1 S$. Hence, the structure of $\mathbf{2}$ was established and named as $(3 R, 19 R)$-19-hydroxy-3-(2-oxopropyl)voacangine.

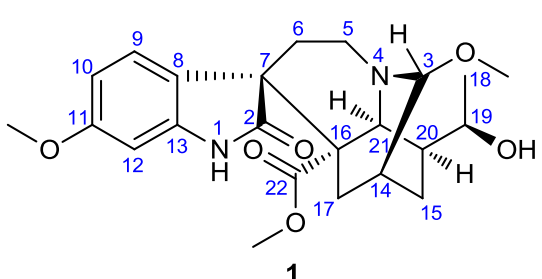

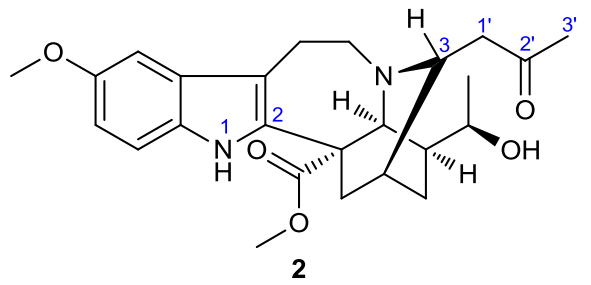

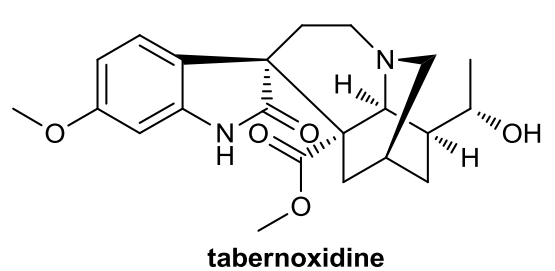

Fig. 1 The structures of compounds $\mathbf{1 , 2}$ and tabernoxidine 
Table $1{ }^{1} \mathrm{H}$ and ${ }^{13} \mathrm{C}$ NMR spectroscopic data of compounds $\mathbf{1}$ and $\mathbf{2}$ in $\mathrm{CDCl}_{3}$ $\left(\delta_{\mathrm{H}} 7.26, \delta_{\mathrm{C}} 77.0 \mathrm{ppm}\right)$

\begin{tabular}{|c|c|c|c|c|}
\hline \multirow[t]{2}{*}{ No. } & \multicolumn{2}{|l|}{1} & \multicolumn{2}{|l|}{2} \\
\hline & $\delta_{\mathrm{H}}$ & $\delta_{\mathrm{C}}$ & $\delta_{\mathrm{H}}$ & $\delta_{\mathrm{C}}$ \\
\hline 1 & $7.90(\mathrm{~s})$ & & $7.70(\mathrm{~s})$ & \\
\hline 2 & & $179.2(\mathrm{~s})$ & & $136.3(\mathrm{~s})$ \\
\hline 3 & $4.18(\mathrm{~d}, 3.3)$ & $91.5(\mathrm{~d})$ & $3.38(\mathrm{dd}, 8.6,4.0)$ & $54.2(\mathrm{~d})$ \\
\hline 5 & $\begin{array}{l}3.46\left(\mathrm{ddd}, 14.6,14.2,3.5, \mathrm{H}_{\alpha}\right) \\
3.05\left(\mathrm{br} \mathrm{dd}, 14.6,5.1, \mathrm{H}_{\beta}\right)\end{array}$ & $48.7(\mathrm{t})$ & $\begin{array}{l}3.34\left(\mathrm{~m}, \mathrm{H}_{\alpha}\right) \\
3.15\left(\mathrm{~m}, \mathrm{H}_{\beta}\right)\end{array}$ & $50.4(\mathrm{t})$ \\
\hline 6 & $\begin{array}{l}2.39\left(\mathrm{td}, 14.2,5.1, \mathrm{H}_{\beta}\right) \\
1.28\left(\mathrm{br} \mathrm{dd}, 14.2,3.5, \mathrm{H}_{\alpha}\right)\end{array}$ & $26.2(\mathrm{t})$ & $\begin{array}{l}3.14\left(\mathrm{~m}, \mathrm{H}_{\beta}\right) \\
3.04\left(\mathrm{~m}, \mathrm{H}_{\alpha}\right)\end{array}$ & $21.7(\mathrm{t})$ \\
\hline 7 & & $50.6(\mathrm{~s})$ & & $109.5(\mathrm{~s})$ \\
\hline 8 & & $123.6(\mathrm{~s})$ & & $128.9(\mathrm{~s})$ \\
\hline 9 & $7.63(\mathrm{~d}, 8.5)$ & $126.9(\mathrm{~d})$ & $6.89(\mathrm{~d}, 2.4)$ & $100.7(d)$ \\
\hline 10 & $6.54(\mathrm{dd}, 8.5,2.6)$ & $106.5(\mathrm{~d})$ & & $154.1(\mathrm{~s})$ \\
\hline 11 & & $160.0(\mathrm{~s})$ & $6.83(\mathrm{dd}, 8.7,2.4)$ & $112.4(\mathrm{~d})$ \\
\hline 12 & $6.43(\mathrm{~d}, 2.6)$ & $97.0(d)$ & $7.15(\mathrm{~d}, 8.7)$ & $111.2(\mathrm{~d})$ \\
\hline 13 & & $140.7(\mathrm{~s})$ & & $130.6(\mathrm{~s})$ \\
\hline 14 & $2.16(\mathrm{~m})$ & $30.1(\mathrm{~d})$ & 1.86 (br s) & $30.5(d)$ \\
\hline 15 & $\begin{array}{l}1.64\left(\mathrm{~m}, \mathrm{H}_{\beta}\right) \\
1.32\left(\mathrm{~m}, \mathrm{H}_{\alpha}\right)\end{array}$ & $21.4(\mathrm{t})$ & $\begin{array}{l}1.90\left(\mathrm{~m}, \mathrm{H}_{\beta}\right) \\
1.63\left(\mathrm{~m}, \mathrm{H}_{\alpha}\right)\end{array}$ & $24.0(\mathrm{t})$ \\
\hline 16 & & $48.7(\mathrm{~s})$ & & $53.7(\mathrm{~s})$ \\
\hline 17 & $\begin{array}{l}3.18\left(\mathrm{~m}, \mathrm{H}_{\beta}\right) \\
1.96\left(\mathrm{dd}, 15.8,2.9, \mathrm{H}_{\alpha}\right)\end{array}$ & $27.2(\mathrm{t})$ & $\begin{array}{l}2.63\left(\mathrm{dd}, 13.8,1.8, \mathrm{H}_{\alpha}\right) \\
2.09\left(\mathrm{ddd}, 13.8,4.0,3.0, \mathrm{H}_{\beta}\right)\end{array}$ & $37.7(\mathrm{t})$ \\
\hline 18 & $1.24(\mathrm{~d}, 6.4)$ & $21.9(q)$ & $1.28(\mathrm{~d}, 6.5)$ & $22.2(q)$ \\
\hline 19 & $3.95(\mathrm{~m})$ & $70.2(\mathrm{~d})$ & $3.89(\mathrm{qd}, 6.5,2.0)$ & $71.0(d)$ \\
\hline 20 & $1.84(\mathrm{~m})$ & $39.2(\mathrm{~d})$ & $1.35(\mathrm{~m})$ & 39.7 (d) \\
\hline 21 & 3.95 (br s) & $50.4(\mathrm{~d})$ & $4.10(\mathrm{~s})$ & $55.2(\mathrm{~d})$ \\
\hline 22 & & $174.3(\mathrm{~s})$ & & $174.7(\mathrm{~s})$ \\
\hline 3-OMe & $3.33(\mathrm{~s})$ & $53.7(q)$ & & \\
\hline $10-\mathrm{OMe}$ & & & $3.84(\mathrm{~s})$ & $56.0(q)$ \\
\hline 11-OMe & $3.78(\mathrm{~s})$ & $55.4(q)$ & & \\
\hline $22-\mathrm{OMe}$ & $3.19(\mathrm{~s})$ & $51.4(q)$ & $3.72(\mathrm{~s})$ & $52.9(\mathrm{q})$ \\
\hline $1^{\prime}$ & & & $\begin{array}{l}2.77(\mathrm{dd}, 17.3,8.6) \\
2.69(\mathrm{dd}, 17.3,4.0)\end{array}$ & $45.1(\mathrm{t})$ \\
\hline $2^{\prime}$ & & & & $207.6(\mathrm{~s})$ \\
\hline $3^{\prime}$ & & & $2.13(\mathrm{~s})$ & $31.0(q)$ \\
\hline
\end{tabular}

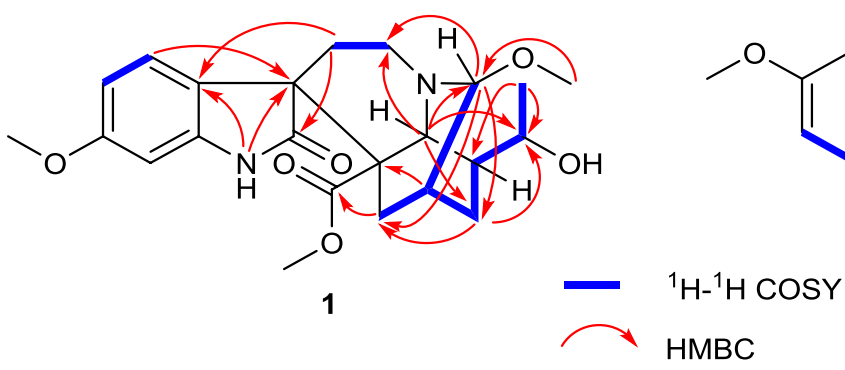

and HMBC ( $)$ correlations of $\mathbf{1}$ and $\mathbf{2}$ 

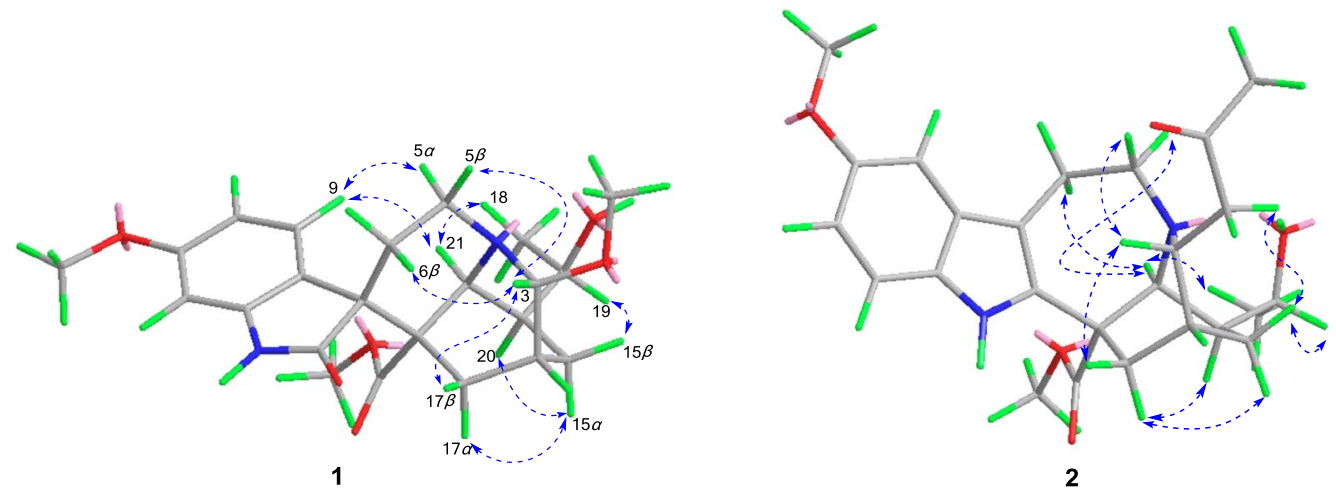

Fig. 3 Key ROESY $(\cdots \cdot \cdot)$ correlations of $\mathbf{1}$ and $\mathbf{2}$

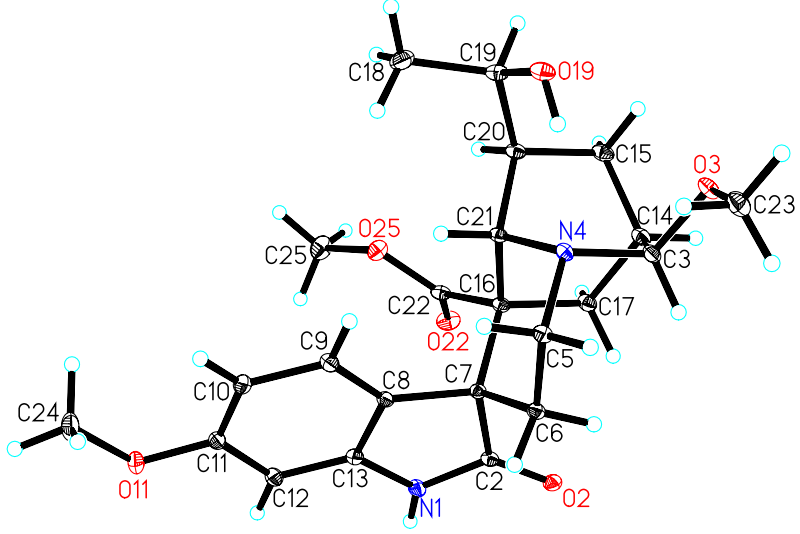

Fig. 4 X-ray crystallographic structure of compound 1

\section{Experimental Section}

\subsection{General Experimental Procedures}

X-ray data were collected using a Bruker APEX DUO diffractometer with graphite-monochromated Mo $\mathrm{K} \alpha$ radiation. Optical rotations were measured on a Rudolph Autopol VI automatic polarimeter. UV data were obtained from HPLC online analysis. IR spectra $(\mathrm{KBr})$ were obtained on a Thermo Nicolet iS10 FT-IR spectrometer. NMR spectra were carried out on a Bruker Avance III 600 or DRX-500 spectrometer with deuterated solvent signals as internal standards. ESIMS and HRESIMS were measured using an Agilent G6230 time-of-flight mass spectrometer. Preparative HPLC separation was performed using an Agilent 1260 series HPLC system equipped with a Zorbax SB-C ${ }_{18}$ column $(5 \mu \mathrm{m}, 21.2 \times 150 \mathrm{~mm})$. Silica gel (200-300 mesh, Qingdao Marine Chemical Inc., China), Chromatorex $\mathrm{C}_{18}(40-75 \mu \mathrm{m}$, Fuji Silysia Chemical Ltd., Japan) and Sephadex LH-20 (GE Healthcare Bio-Sciences AB, Uppsala, Sweden) were used for column chromatography. Fractions were monitored and analyzed using TLC, in combination with Agilent 1200 series HPLC system equipped by an Extend- $\mathrm{C}_{18}$ column $(5 \mu \mathrm{m}, 4.6 \times 150 \mathrm{~mm})$.

\subsection{Plant Material}

The twigs and leaves of Tabernaemontana divaricata were collected from Menglun town, Xishuangbanna Dai Autonomous Prefecture in Yunnan Province, China, in January 2014 and identified by Mr. Yu Chen of Kunming Institute of Botany, Chinese Academy of Sciences. A voucher specimen (No. BBP0671) was deposited at BioBioPha Co., Ltd.

\subsection{Extraction and Isolation}

The twigs and leaves of $T$. divaricata were extracted with 95\% aqueous $\mathrm{EtOH}$ at room temperature, and the solvent was removed under reduced pressure to give crude extract ( $c a$. $600 \mathrm{~g}$ ), then dissolved in $2 \% \mathrm{HCl}$ and filtered. The filtration was basified using $10 \%$ ammonia-water to $\mathrm{pH} 9-10$ and then partitioned with EtOAc to give a total alkaloidal extract ( $c a$. $100 \mathrm{~g}$ ), which was fractionated by silica gel CC successively eluted with $\mathrm{CHCl}_{3} / \mathrm{MeOH}(200: 1 \rightarrow 10: 1$ gradient) to give six fractions $\mathrm{A}-\mathrm{E}$, respectively. Fraction $\mathrm{B}$ was separated by silica gel $\mathrm{CC}\left(\mathrm{CHCl}_{3} / \mathrm{MeOH}, 100: 1 \rightarrow 80: 1\right)$ into fractions $\mathrm{B}_{1}-\mathrm{B}_{4}$. Fraction $\mathrm{B}_{1}$ was purified by silica gel $\mathrm{CC}$ using petroleum ether (PE)/acetone (4:1) and Sephadex LH-20 $\left(\mathrm{CHCl}_{3} / \mathrm{MeOH}, 1: 1\right)$ to obtain $7(50 \mathrm{mg})$. Fraction $\mathrm{B}_{2}$ was separated by silica gel $\mathrm{CC}\left(\mathrm{CHCl}_{3} / \mathrm{MeOH}, 100: 1\right)$ to give 8 $(18 \mathrm{mg})$ and $\mathbf{9}(4 \mathrm{mg})$. Fraction $\mathrm{C}$ was separated by silica gel $\left(\mathrm{CHCl}_{3} / \mathrm{MeOH}, 100: 1 \rightarrow 60: 1\right)$ into fractions $\mathrm{C}_{1}-\mathrm{C}_{3}$. Fraction $\mathrm{C}_{1}$ was separated by Sephadex LH-20 $\left(\mathrm{CHCl}_{3} / \mathrm{MeOH}, 1: 1\right)$ to obtain compound $5(17 \mathrm{mg})$. Fraction $\mathrm{C}_{2}$ was separated by silica gel $\left(\mathrm{CHCl}_{3} / \mathrm{MeOH}, 60: 1\right)$, prep-HPLC $\left(\mathrm{MeOH} / \mathrm{H}_{2} \mathrm{O}\right.$; $60 \%)$ and Sephadex $\mathrm{LH}-20\left(\mathrm{CHCl}_{3} / \mathrm{MeOH}, 1: 1\right)$ to obtain compounds $\mathbf{1}(46 \mathrm{mg}), \mathbf{4}(3 \mathrm{mg})$ and $\mathbf{1 0}(102 \mathrm{mg})$. Fraction D was separated by silica gel $\mathrm{CC}\left(\mathrm{CHCl}_{3} / \mathrm{MeOH}, 60: 1 \rightarrow 30: 1\right)$ 
into fractions $\mathrm{D}_{1}-\mathrm{D}_{4}$. Fraction $\mathrm{D}_{2}$ was further purified by silica gel $\left(\mathrm{CHCl}_{3} / \mathrm{MeOH}, 80: 1\right)$ and prep-HPLC $(\mathrm{MeOH} /$ $\left.\mathrm{H}_{2} \mathrm{O} ; 80 \%\right)$ to give $2(14 \mathrm{mg})$. Fraction $\mathrm{E}$ was subjected to MPLC $\left(\mathrm{MeOH} / \mathrm{H}_{2} \mathrm{O}, 50 \%\right)$ and then by Sephadex LH-20 $(\mathrm{MeOH})$ to give $3(33 \mathrm{mg})$ and $\mathbf{6}(13 \mathrm{mg})$. The retention times $\left(t_{\mathrm{R}}\right)$ of $\mathbf{1}$ and $\mathbf{2}$ on an analytical HPLC InertSustain $\mathrm{C}_{\mathbf{1 8}}$ column $\left(20 \% \rightarrow 100 \% \mathrm{MeOH}\right.$ in $\mathrm{H}_{2} \mathrm{O}$ over 8.0 min followed by $100 \% \mathrm{MeOH}$ to $13.0 \mathrm{~min}, 1.0 \mathrm{ml} / \mathrm{min}$ ) were 4.60 and $6.00 \mathrm{~min}$, respectively.

\section{4 (3R)-7,19-Di-epi-3-methoxytabernoxidine (1)}

Colorless block crystals; $[\alpha]_{\mathrm{D}}^{22}+25.9(c 0.10, \mathrm{MeOH})$; UV $(\mathrm{MeOH}) \lambda_{\text {max }}: 217,261,285$ (sh) nm; IR (KBr) $\nu_{\max }: 3427$, 2966, 2945, 2936, 2901, 2835, 1720, 1631, 1594, 1507, $1468,1365,1314,1197,1160,1101,829 \mathrm{~cm}^{-1} ;{ }^{1} \mathrm{H}$ and ${ }^{13} \mathrm{C}$ NMR data: see Table 1; ESIMS (pos.): $m / z 453[\mathrm{M}+\mathrm{Na}]^{+}$; HRESIMS (pos.): $\mathrm{m} / \mathrm{z} 453.1997[\mathrm{M}+\mathrm{Na}]^{+}$(calcd. for $\mathrm{C}_{23} \mathrm{H}_{30} \mathrm{~N}_{2} \mathrm{O}_{6} \mathrm{Na}, 453.1996$ ).

\subsection{Crystallographic Data for 1}

$\mathrm{C}_{23} \mathrm{H}_{30} \mathrm{~N}_{2} \mathrm{O}_{6} \cdot \mathrm{CH}_{4} \mathrm{O}, \quad M=462.53$, monoclinic, $a=10.9955(11) \AA, b=6.7753(7) \AA, c=15.2342(15)$ $\AA, \alpha=90.00^{\circ}, \beta=92.975(2)^{\circ}, \gamma=90.00^{\circ}, V=1133.4(2)$ $\AA^{3}, \quad T=100(2) \quad \mathrm{K}$, space group $P 21, \quad Z=2$, $\mu(\mathrm{MoK} \alpha)=0.100 \mathrm{~mm}^{-1}, 12217$ reflections measured, 6280 independent reflections $\left(R_{\text {int }}=0.0225\right)$. The final $R_{l}$ values were $0.0339(I>2 \sigma(I))$. The final $w R\left(F^{2}\right)$ values were 0.0866 $(I>2 \sigma(I))$. The final $R_{l}$ values were 0.0385 (all data). The final $w R\left(F^{2}\right)$ values were 0.0893 (all data). The goodness of fit on $F^{2}$ was 1.043. Flack parameter $=0.3(3)$.

\section{6 (3R,19R)-19-Hydroxy-3-(2-oxopropyl) voacangine (2)}

White amorphous powder; $[\alpha]_{\mathrm{D}}^{22}-39.3(c 0.10, \mathrm{MeOH})$; $\mathrm{UV}(\mathrm{MeOH}) \lambda_{\text {max }}: 223,287 \mathrm{~nm}$; IR (KBr) $\nu_{\text {max }}: 3442,2934$, 2871, 1711, 1627, 1487, 1455, 1225, 1166, 1077, 1033, 828, $798 \mathrm{~cm}^{-1} ;{ }^{1} \mathrm{H}$ and ${ }^{13} \mathrm{C}$ NMR data: see Table 1; ESIMS (pos.): $m / z 441[\mathrm{M}+\mathrm{H}]^{+}$; HRESIMS (pos.): $m / z 441.2384[\mathrm{M}+\mathrm{H}]^{+}$ (calcd. for $\mathrm{C}_{25} \mathrm{H}_{33} \mathrm{~N}_{2} \mathrm{O}_{5}, 441.2384$ ).

Acknowledgements This work was financially supported by the "Large-scale Compound Library" project of National Development and Reform Commission of China.

\section{Compliance with Ethical Standards}

Conflict of interest The authors declare no conflict of interest.

Open Access This article is distributed under the terms of the Creative Commons Attribution 4.0 International License (http://creativeco mmons.org/licenses/by/4.0/), which permits unrestricted use, distribution, and reproduction in any medium, provided you give appropriate credit to the original author(s) and the source, provide a link to the Creative Commons license, and indicate if changes were made.

\section{References}

1. M.T. Andrade, J.A. Lima, A.C. Pinto, C.M. Rezende, M.P. Carvalho, R.A. Epifanio, Bioorg. Med. Chem. 13, 4092-4095 (2005)

2. H. Achenbach, M. Benirschke, R. Torrenegra, Phytochemistry 45, 325-335 (1997)

3. T.A. Van Beek, R. Verpoorte, A.B. Svendsen, R. Fokkens, J. Nat. Prod. 48, 400-423 (1985)

4. Editorial Committee of Flora Reipublicae Popularis Sinicae, Flora Reipublicae Popularis Sinicae. Academic Press: Beijing 63, 106 (1977)

5. Z.X. Zhu, H.S. Chen, J. Pharm. Pract. 29, 412-415 (2011)

6. M.F. Bao, J.M. Yan, G.G. Cheng, X.Y. Li, Y.P. Liu, Y. Li, X.H. Cai, X.D. Dong, J. Nat. Prod. 76, 1406-1412 (2013)

7. T.S. Kam, H.S. Pang, Y.M. Choo, K. Komiyama, Chem. Biodivers. 1, 646-656 (2004)

8. M. Zeches, T. Ravao, B. Richard, G. Massiot, L. Le Men-Olivier, R. Verpoorte, J. Nat. Prod. 50, 714-720 (1987)

9. Atta-ur-Rahman, K.A. Alvi, S.A. Abbas, W. Voelter, Heterocycles 26, 413-419 (1987)

10. P. Clivio, B. Richard, J.R. Deverre, T. Sevenet, M. Zeches, L. Le Men-Oliver, Phytochemistry 30, 3785-3792 (1991)

11. T.S. Kam, K.Y. Loh, C. Wei, J. Nat. Prod. 56, 1865-1871 (1993)

12. B.J. Zhang, X.F. Teng, M.F. Bao, X.H. Zhong, L. Ni, X.H. Cai, Phytochemistry 120, 46-52 (2015)

13. W.L.B. Medeiros, I.J.C. Vieira, L. Mathias, R. Braz-Filho, K.Z. Leal, E. Rodrigues-Filho, J. Schripsema, Magn. Reson. Chem. 37, 676-681 (1999)

14. J. Éles, G. Kalaus, I. Greiner, M. Kajtár-Peredy, P. Szabó, G.M. Keserû, L. Szabó, C. Szántay, J. Org. Chem. 67, 7255-7260 (2002)

15. T.S. Kam, S. Anuradha, K.Y. Loh, Nat. Prod. Lett. 8, 49-53 (1996)

16. V.S.P. Chaturvedula, S. Sprague, J.K. Schilling, D.G.I. Kingston, J. Nat. Prod. 66, 528-531 (2003)

17. B.S. Joshi, P.G. Rao, D. Rogers, B.P. Singri, D.J. Williams, Indian J. Chem. 23B, 101-102 (1984)

18. E. Okuyama, L.H. Gao, M. Yamazaki, Chem. Pharm. Bull. 40, 2075-2079 (1992)

19. P. Perera, F. Sandberg, T.A. van Beek, R. Verpoorte, Phytochemistry 24, 2097-2104 (1985)

20. T.S. Kam, K.M. Sim, J. Nat. Prod. 65, 669-672 (2002) 